\title{
Avaliação nutricional de pacientes em hemodiálise
}

\author{
Nutritional evaluation of patients on hemodialysis
}

Poliana Coelho CABRAL

Alcides da Silva DINIZ²

Ilma Kruze Grande de ARRUDA²

\section{R E S U M O}

\section{Objetivo}

O objetivo deste estudo foi o de avaliar o estado nutricional e a ingestão de energia e de nutrientes de uma população em hemodiálise no Hospital das Clínicas, Universidade Federal de Pernambuco.

\section{Métodos}

De um total de 47 pacientes em hemodiálise de manutenção, 37 indivíduos (18 homens e 19 mulheres, idade 50,4 $\pm 16,3$ anos) foram selecionados. O índice de massa corporal foi utilizado para a classificação do estado nutricional e a dieta foi investigada por meio do método do diário alimentar de 4 dias.

\section{Resultados}

Os resultados evidenciaram um predomínio de indivíduos eutróficos (62,2\%) e igual prevalência de baixo peso e excesso de peso (18,9\% de pacientes em cada caso). Com relação à dieta, os achados desta pesquisa revelaram um adequado consumo energético-protéico. De uma forma geral, a ingestão média diária de nutrientes foi considerada adequada, exceto pelo cálcio e pela vitamina A, que apresentaram $<50,0 \%$ e $<70,0 \%$ da ingestão diária recomendada, respectivamente.

\section{Conclusão}

Esses resultados indicam que se deve dar atenção cuidadosa às diferenças regionais e nacionais que influenciam o estado nutricional e a ingestão dietética de pacientes em hemodiálise.

Termos de indexação: antropometria, consumo de alimentos, diálise renal, dietética, doença renal crônica.

\footnotetext{
1 Doutoranda em Nutrição, Programa de Pós-Graduação em Nutrição; Departamento de Nutrição, Laboratório de Nutrição Clínica, Universidade Federal de Pernambuco. Av. Moraes Rego, s/n., Cidade Universitária, 50670-420, Recife, PE, Brasil. Correspondência para/Correspondence to: P.C. CABRAL. E-mail: <pccabral@uol.com.br>.

2 Departamento de Nutrição, Laboratório de Nutrição Clínica, Universidade Federal de Pernambuco. Recife, PE, Brasil.
} 


\section{A B S T R A C T}

\section{Objective}

To evaluate the nutritional status, and the energy and nutrient intakes of a population undergoing hemodialysis at the Hospital das Clínicas, Universidade Federal de Pernambuco, Brazil.

\section{Methods}

From a pool of 47 outpatients undergoing maintenance hemodialysis, 37 individuals (18 males and 19 females, aged $50.4 \pm 16.3$ years) were selected. The body-mass index was used to determine the nutritional status, and the diet was investigated through the 4-day food diary method.

\section{Results}

The results revealed a predominance of normal, eutrophic patients (62.2\%), while presenting equal prevalence of underweight and overweight patients (18.9\% of them in each case). Regarding the diet, the findings of this survey showed there was adequate protein-energy consumption. In general, the average daily intake of nutrients was adequate, except for calcium and vitamin $A$, which presented only $<50,0 \%$ and $<70,0 \%$, respectively, of the recommended daily intake.

\section{Conclusion}

These results indicate that careful attention should be paid to regional and national differences when considering the nutritional status and dietary intake of hemodialysis patients.

Indexing terms: anthropometry, food consumption, renal dialysis, dietetics, chronic renal disease.

\section{N T R O D U Ç Ã O}

A insuficiência renal crônica (IRC) pode ser definida como uma síndrome complexa, que se caracteriza pela perda lenta, progressiva e irreversível das funções renais ${ }^{1}$.

No Brasil, ainda não existem dados confiáveis que possam retratar a incidência e a prevalência da IRC. Segundo Lessa ${ }^{2}$, isso resulta da dificuldade em obter um diagnóstico precoce e, conseqüentemente, os estudos existentes se baseiam em indivíduos com doença renal avançada, já inseridos em programas de diálise.

Em 2002, estimava-se em 54523 pacientes a população em diálise no Brasil, cerca de 19\% desse total (10 285) encontrava-se na Região Nordeste ${ }^{3}$.

Apesar dos avanços no tratamento da IRC, a morbi-mortalidade continua elevada: no Brasil, a sobrevida gira em torno de $79 \%$ e $41 \%$, respectivamente no primeiro e no quinto ano de diálise ${ }^{4}$, sendo que a desnutrição protéico-energética (DPE), é um importante fator que contribui para esse quadro nosológico. Levantamentos mostram que, em todo o mundo, $6 \%$ a $8 \%$ dos indivíduos submetidos a tratamento dialítico sofrem de desnutrição grave, e cerca de $33 \%$ de desnutrição leve a moderada ${ }^{5-7}$.

Inúmeras são as razões para a desnutrição em pacientes em diálise, incluindo distúrbios no metabolismo protéico e energético, alterações hormonais e ingestão alimentar deficiente, devidos, principalmente a anorexia, náuseas e vômitos, manifestações clínicas freqüentes no estado de toxicidade urêmica ${ }^{7}$.

Desse modo, o reconhecimento da desnutrição, como importante fator de risco no nefropata crônico fez com que proliferassem, na literatura especializada, pesquisas sobre o tema. No entanto, no Nordeste brasileiro, as informações ainda são bastante escassas, principalmente no que se refere ao consumo alimentar. 
O objetivo principal desse trabalho foi o de descrever e analisar algumas características antropométricas, bioquímicas e dietéticas de pacientes cadastrados junto ao programa de hemodiálise regular do Hospital das Clínicas (HC) da Universidade Federal de Pernambuco (UFPE), visando obter subsídios para uma orientação nutricional mais adequada.

\section{CASUÍSTICA E MÉTODOS}

Esse estudo, do tipo série de casos, foi realizado no Serviço de Nefrologia do Hospital das Clínicas, Universidade Federal de Pernambuco (HC/UFPE), que possui, inseridos no programa de hemodiálise regular, 47 pacientes. Para compor a amostra foram selecionados os indivíduos de acordo com os seguintes critérios de elegibilidade: pertencer ao programa de hemodiálise regular do HC/UFPE; ter idade entre 20 e 80 anos; não ser portador de nenhum outro tipo de doença crônica e consumptiva. Com base nos parâmetros citados, o grupo amostral foi constituído por 37 pacientes, correspondendo a 78,7\% da população em diálise, no referido hospital.

O estado nutricional foi avaliado, utilizando-se o índice de massa corporal (IMC), indicador reconhecido e clinicamente útil na avaliação de pacientes renais crônicos ${ }^{8}$. Como o estado de hidratação pode influenciar significativamente essa avaliação, foi utilizado o "Peso Seco", ou seja, aquele que é observado pós-hemodiálise. A classificação utilizada foi a proposta pela Organização Mundial de Saúde, em $1995^{9}$.

Foram analisados o índice de remoção de uréia (Kt/V), para avaliar a adequação do procedimento dialítico, e as concentrações de albumina sérica, classificando-se ambos de acordo com os pontos de corte apresentados por Martins \& Riella7. A fórmula utilizada para o cálculo do KtN (Daugirdas II), bem como o método analítico empregado para a determinação da albumina sérica (verde de bromcresol), também encontram-se descritos em Martins \& Riella7.
Existem vários métodos para estimar o consumo de nutrientes, sendo que os mais utilizados são o Recordatório de 24 horas e o Auto-registro ou Diário alimentar ${ }^{10}$. Esta pesquisa utilizou o Diário alimentar, no qual os pacientes registraram seu consumo por um período de 4 dias, assim distribuídos: 1 dia do final de semana, 2 no período interdialítico e 1 dia de diálise. Todos foram orientados por um nutricionista para a realização do diário; os indivíduos analfabetos tiveram o diário preenchido por um familiar responsável. O registro foi feito em medidas caseiras e a conversão destas em gramas foi feita posteriormente, utilizando-se como padrão de referência a tabela de Pinheiro et al. ${ }^{11}$.

Os resultados obtidos foram comparados às recomendações específicas para pacientes renais em hemodiálise ${ }^{7,12}$ e adultos normais ${ }^{13}$. No que se refere às recomendações de energia e proteínas, os valores de referência utilizados para o tratamento dialítico ${ }^{7}$ (25 a 45 kcal/kg/dia e 1,2 a 1,4g de proteína/kg/dia) foram estipulados de acordo com a necessidade de aquisição ou manutenção de um estado nutricional adequado. Quanto às vitaminas e aos oligoelementos, utilizaram-se como padrões as recomendações específicas para pacientes renais em hemodiálise ${ }^{12}$, cuja elaboração levou em conta as perdas ou acúmulos ocorridos durante o tratamento. Por outro lado, devido à inexistência, na literatura especializada, de recomendação para a vitamina A em pacientes submetidos a diálise, foi utilizado o valor recomendado para adultos saudáveis ${ }^{13}$.

A construção do banco de dados e a análise estatística foram realizadas no programa Epi Info versão 6.04 ${ }^{14}$. Com o objetivo de avaliar o comportamento das variáveis segundo o critério de normalidade da distribuição, utilizou-se o programa estatístico SPSS ${ }^{15}$.

Na descrição das proporções, a distribuição binomial foi aproximada à distribuição normal pelo intervalo de confiança de 95\%. A comparação entre as médias foi realizada, ou pelo teste " $\mathrm{t}$ " de Student (duas médias), ou pela Análise de Variância (mais de duas médias), utilizando-se o 
teste de Scheffé a posteriori, quando necessário. Quando os critérios paramétricos (distribuição normal e homocedasticidade) não foram atingidos, os testes não paramétricos de Mann Whitney e Kruskal-Wallis foram utilizados. Foi adotado o nível de significância de 5\% para rejeição da hipótese de nulidade.

A análise da composição da dieta foi realizada por meio do software de apoio à Nutrição, da Escola Paulista de Medicina ${ }^{16}$. A tabela base deste programa é a do Departamento de Agricultura dos Estados Unidos, ano 1976-1986. Desse modo, em virtude da ocorrência de inúmeros produtos de consumo regional, alguns alimentos tiveram que ser acrescidos, sendo utilizada a Tabela de Franco ${ }^{17}$.

O protocolo de pesquisa foi aprovado pelo comitê de ética do Instituto Materno Infantil de Pernambuco (IMIP), de acordo com as normas para pesquisas em seres humanos.

\section{RESULTADOSE DISCUSSÃ O}

Conforme observado (Tabela 1), houve uma distribuição homogênea dos pacientes, no que diz respeito ao sexo e à idade, considerando-se os respectivos intervalos de classe.

Analisando a situação em termos de médias (Tabela 2), verifica-se que a faixa etária neste estudo ficou próxima aos 50 anos, retratando um grupo relativamente jovem, se comparado aos pacientes europeus, cuja média de idade oscila entre 58 e 62 anos $^{18}$.

A baixa freqüência de idosos em tratamento dialítico no país é típica de regiões pobres, onde, apesar da incidência crescente das doenças crônicas não transmissíveis, as principais causas de IRC continuam sendo os processos infecciosos (como a glomerulonefrite crônica) que afetam populações mais jovens, quadro bem diferente do que ocorre em regiões mais desenvolvidas ${ }^{1}$.

Tabela 1. Características socioeconômicas e demográficas de pacientes submetidos a hemodiálise regular no Hospital das Clínicas. Recife, 2002.

\begin{tabular}{|c|c|c|c|}
\hline Variáveis & $n=37$ & $\%$ & IC $95 \% *$ \\
\hline \multicolumn{4}{|l|}{ Sexo } \\
\hline Masculino & 18 & 48,6 & $32,2-65,3$ \\
\hline Feminino & 19 & 51,4 & $34,7-67,8$ \\
\hline \multicolumn{4}{|l|}{ Idade (anos) } \\
\hline $20-44$ & 12 & 32,4 & $18,6-49,9$ \\
\hline $45-59$ & 14 & 37,8 & $22,9-55,2$ \\
\hline$\geq 60$ & 11 & 29,7 & $16,4-47,2$ \\
\hline \multicolumn{4}{|l|}{ Instrução } \\
\hline Analfabeto & 15 & 40,6 & $25,2-57,8$ \\
\hline$\leq 4^{a}$ série - 1ํ Grau & 12 & 32,4 & $18,6-49,9$ \\
\hline$\geq 5^{a}$ série - 1ํ Grau & 10 & 27,0 & $14,4-44,4$ \\
\hline \multicolumn{4}{|l|}{ Ocupação } \\
\hline Aposentado & 32 & 88,5 & $70,4-94,9$ \\
\hline Outra & 5 & 13,5 & $5,1-29,6$ \\
\hline \multicolumn{4}{|c|}{ Renda Familiar Mensal } \\
\hline$\leq 2$ salários mínimos & 31 & 83,8 & $67,3-93,2$ \\
\hline > 2 salários mínimos & 6 & 16,2 & $6,8-32,7$ \\
\hline \multicolumn{4}{|c|}{ Número de pessoas no domicilio** } \\
\hline$\leq 4$ & 23 & 63,9 & $46,2-78,7$ \\
\hline$>4$ & 13 & 36,1 & $21,3-53,8$ \\
\hline
\end{tabular}

* IC = Intervalo de Confiança ; ** 1 paciente residia em um hospital psiquiátrico. 
Tabela 2. Dados antropométricos, bioquímicos e tempo de tratamento, de pacientes submetidos à hemodiálise regular no Hospital das Clínicas. Recife, 2002.

\begin{tabular}{|c|c|c|c|c|}
\hline \multirow{2}{*}{ Vexo } & \multicolumn{2}{|c|}{ Homens $(n=18)$} & Mulheres $(n=19)$ & \multirow{2}{*}{ Valor de $p^{* * *}$} \\
\hline & $x$ & DP & DP & \\
\hline Idade (anos) & $51,90 \pm$ & 17,00 & $48,90 \pm 15,90$ & 0,465 \\
\hline Altura (m) & $1,60 \pm$ & 0,11 & $1,55 \pm 0,04$ & $0,003 \bullet$ \\
\hline Peso (kg) & $56,50 \pm$ & 12,00 & $51,80 \pm 10,70$ & 0,218 \\
\hline IMC $\left(\mathrm{kg} / \mathrm{m}^{2}\right)$ & $22,00 \pm$ & 3,30 & $21,90 \pm 4,36$ & 0,954 \\
\hline Tempo de HD* (meses) & $45,70 \pm$ & 61,70 & $20,10 \pm 18,50$ & $0,241 \bullet$ \\
\hline $\mathrm{Kt} / \mathrm{N}^{* *}$ & $1,46 \pm$ & 0,23 & $1,63 \pm 0,30$ & 0,092 \\
\hline Albumina sérica ( $\mathrm{g} / \mathrm{dL}$ ) & $3,30 \pm$ & 0,40 & $3,30 \pm 0,40$ & \\
\hline
\end{tabular}

*HD= Hemodiálise; **KtN= Um dos índices de remoção de uréia; ***Teste t de Student; • Kruskal-Wallis; • p< 0,05.

Outro dado importante (Tabela 1) é o precário nível socioeconômico do grupo em estudo, tendo em vista que 83,8\% das famílias subsistiam com renda igual ou inferior a 2 salários mínimos, com $40,6 \%$ dos pacientes declarando-se sem instrução. Estas condições desfavoráveis provavelmente interferem na compreensão e na adesão ao tratamento proposto.

É notável a informação obtida (Tabela 2), de que, apesar das precárias condições socioeconômicas dos pacientes, a média do IMC se situou dentro da faixa de normalidade. Com respeito a isso, os dados (Tabela 3), especificando a distribuição dos resultados segundo os pontos de corte do IMC, demonstram uma incidência de baixo peso ou magreza em 18,9\% (IC 95,0\% 8,6 - 35,7) dos pacientes. Utilizando o mesmo indicador nutricional (IMC), pesquisas realizadas no Brasil ${ }^{19}$ e no exterior ${ }^{20,21}$ relataram prevalências de baixo peso em 4,0\% e em $20,0 \%$ dos pacientes, respectivamente. Esta variação deve-se, provavelmente, às diferenças nas populações estudadas (faixa etária, por exemplo), e aos dois pontos de corte utilizados na classificação do baixo peso (IMC $<18,5^{19}$ ou IMC $<20,020,21)$. Valenzuela et al. ${ }^{19}$, em seu estudo com pacientes no Amazonas/Brasil, apesar da ocorrência de baixo peso em apenas 4,0\% dos pacientes, evidenciaram desnutrição em 44,8\% deles, quando os parâmetros utilizados foram as medidas do braço: da prega cutânea tricipital
(PCT) e da circunferência muscular do braço (CMB). Segundo os pesquisadores sugeriram, a falta de repercussão da desnutrição sobre o IMC, em parte, poderia ser explicada pelas alterações no estado de hidratação do paciente, cujo peso aumentaria com reflexos menores sobre a PCT e CMB.

Segundo as diretrizes da National Kidney Foundation (K/DOQI) ${ }^{8}$, outras medidas de antropometria, como a percentagem do peso usual, percentagem do peso ideal e o IMC, são mais precisas do que as medidas das pregas cutâneas e da circunferência do braço. No entanto, nenhum indicador, utilizado de forma isolada, é capaz de gerar informações suficientes para uma análise mais profunda do estado nutricional.

Infelizmente, neste estudo, não foram coletadas as medidas do braço, o que enriqueceria a análise dos resultados. Por outro lado, existem evidencias, de que os pacientes em hemodiálise crônica com maior IMC têm maior sobrevida ${ }^{22,23}$, em contraste com a população de um modo geral; isto sugere o uso do IMC, também como indicador de morbi-mortalidade.

Outro parâmetro importante na avaliação nutricional do nefropata crônico é a adequação do procedimento dialítico, uma vez que a diálise inadequada pode resultar em um estado urêmico, conduzindo a náuseas, vômitos e anorexia, com conseqüente prejuízo no consumo alimentar? . 
A adequação da diálise depende de vários fatores, mas tem sido relacionada, principalmente, à dose ou quantidade de diálise oferecida a um paciente; pode-se avaliar essa adequação por meio da medida dos índices de remoção de uréia, como, por exemplo, o Kt/V. A razão Kt/N representa quantas vezes a água corporal de um paciente foi totalmente depurada de um soluto, no caso, a uréia ${ }^{24}$. Atualmente, a recomendação é de um $\mathrm{Kt} / \mathrm{N}$ igual ou superior a 1,3 para a hemodiálise, sendo que, possivelmente, os valores entre 1,4 e 1,6 sejam os melhores ${ }^{7}$.

Os resultados deste estudo (Tabela 2) demonstram que os pacientes foram submetidos a sessões de hemodiálise de boa eficiência ( $\mathrm{KtN}$ de 1,46 e 1,63 para homens e mulheres, respectivamente), com valores superiores aos encontrados nos Estados Unidos (1,26), Japão $(1,37)$ e países europeus $(1,26-1,49)^{18}$. No entanto, um dos principais problemas na utilização do $\mathrm{Kt} / \mathrm{V}$ é a coleta inadequada da uréia pré- e pós-diálise, que pode ocasionar resultados pouco confiáveis ${ }^{24}$. Além disso, Kuhlmann et al. ${ }^{25}$ demonstraram que, pacientes de maior superfície corporal e peso, condições comuns em países desenvolvidos, apresentam grande dificuldade para atingir as metas de $\mathrm{Kt} / \mathrm{N}$.

A albumina sérica, apesar de suas limitações, também pode ser útil na avaliação nutricional do paciente renal crônico ${ }^{7}$. A hipoalbuminemia tem importante papel nas condições do paciente em diálise, pois é um preditor forte e independente de mortalidade ${ }^{26}$. Os estudos mostram que o risco de morte aumenta, acentuadamente, quando os níveis séricos de albumina declinam a valores inferiores a $4 \mathrm{~g} / \mathrm{dL}^{26,27}$.

Neste estudo, o valor médio para a albumina sérica (Tabela 2) foi de 3,30 $\pm 0,40 \mathrm{~g} / \mathrm{dL}$, em que 40,5\% (IC 95,0\% 25,2 - 57,8) dos indivíduos da amostra (Tabela 3) apresentaram valores entre 3,5 a 3,9g/dL; apenas 5,4\% (IC 95\% 0,9 - 19,5) exibiram valores iguais ou superiores a $4,0 \mathrm{~g} / \mathrm{dL}$. Quanto a estes resultados, é importante frisar que a hipoalbuminemia nem sempre reflete apenas déficit nutricional; existem evidências na literatura de que os pacientes com IRC podem apresentar estado inflamatório crônico, o qual se traduz em elevação dos marcadores inflamatórios e redução na síntese hepática de albumina ${ }^{28}$. Desse modo, na ocorrência de inflamação, a albumina, por não ser uma proteína de fase aguda, tem sua síntese prejudicada. Infelizmente, nenhum marcador de inflamação foi avaliado nesta população de renais crônicos, o que poderia ter contribuído para a correta interpretação da hipoalbuminemia apresentada por uma parcela considerável dos pacientes estudados.

Tabela 3. Classificação antropométrica (IMC) e concentrações de albumina sérica dos pacientes submetidos a hemodiálise regular no Hospital das Clínicas. Recife, 2002.

\begin{tabular}{lcrr}
\hline Variável (pontos de corte) & $\mathrm{n}$ & $\%$ & IC $95 \%$ * \\
\hline IMC $\left(\mathrm{kg} / \mathrm{m}^{2}\right)$ & & & $8,6-35,7$ \\
$<18,5$ & 7 & 18,9 & $44,8-77,1$ \\
18,5 a 24,9 & 23 & 62,2 & $6,8-32,7$ \\
25,0 a 29,9 & 6 & 16,2 & $0,1-15,8$ \\
$\geq 30$ & 1 & 2,7 & $37,1-70,2$ \\
Albumina sérica $(\mathrm{g} / \mathrm{dL})$ & & 54,1 & $25,2-57,8$ \\
$<3,5$ & 20 & 40,5 & $0,9-19,5$ \\
3,5 a 3,9 & 15 & 5,4 & \\
$\geq 4,0$ & 2 & & \\
\hline
\end{tabular}

*IC = Intervalo de Confiança. 


\section{AR ACTERÍSTICAS DO CONSUMO ALIMENTAR}

A ingestão deficiente de energia e proteínas tem sido citada como uma das principais causas de desnutrição no tratamento dialítico ${ }^{7}$. Neste estudo, os dados (Tabela 4) demonstram que, independentemente do sexo, a ingestão de energia e proteínas não foi deficitária, apresentando percentuais de adequação superiores a 90\% (energia) e 100\% (proteínas). Em média, os pacientes ingeriram em torno de $31 \mathrm{kcal} / \mathrm{kg} / \mathrm{dia}$ e $1,4 \mathrm{~g}$ de proteína $/ \mathrm{kg} / \mathrm{dia}$, valores dentro do recomendado ${ }^{7}$ e superiores aos encontrados em alguns estudos nacionais ${ }^{19} \mathrm{e}$ internacionais ${ }^{5}$.

Outra informação importante diz respeito à qualidade da proteína consumida, recomendando-se $50 \%$ a $80 \%$ de proteínas de alto valor biológico (AVB), com o objetivo de assegurar o aporte adequado dos aminoácidos essenciais ${ }^{7}$. Conforme pode ser evidenciado (Tabela 4), esse percentual na amostra foi superior a $60 \%$.

Quanto à distribuição percentual, segundo os pontos de corte da adequação dietética, constatou-se que $35,1 \%$ (IC 95,0\% 20,7 - 52,6) e 10,8\% (IC 95,0\% 3,5 - 26,4) dos pacientes apresentaram baixo consumo de calorias e proteínas, respectivamente, revelando um sub-grupo que necessita ser melhor assistido. Vale a pena destacar que, entre os indivíduos com excesso de peso (18,9\% da amostra - Tabela 3), foi determinado, como recomendado, o teor energético já ajustado para a perda ponderal.
Portanto, a redução intencional de calorias da dieta não se constituiu em uma variável de confusão ao estudo.

Outro dado que merece ser comentado é que, em virtude das precárias condições socioeconômicas, os pacientes inscritos no programa de hemodiálise recebem uma cesta básica mensal com oito itens (ovos, leite em pó, arroz, macarrão, feijão, fubá, açúcar e doce) e são orientados por nutricionistas sobre a melhor forma de administrar os escassos recursos disponíveis. Além disso, o serviço de nutrição do HC/UFPE oferece nos três dias de diálise, duas refeições principais e um lanche, todos com elevado teor energético protéico, totalizando $1700 \mathrm{kcal}$ e $90 \mathrm{~g}$ de proteína/dia. Medidas como as citadas acima, devem ter contribuído para o adequado consumo constatado em grande parte dos pacientes analisados.

Deve-se observar que, para uma estimativa mais confiável do consumo do paciente em tratamento dialítico, recomenda-se um diário alimentar de pelo menos três dias, incluindo um dia do final de semana, um do período interdialítico e um dia de diálise. Períodos mais longos, por exemplo sete dias, aumentam a probabilidade de erro devido à queda da motivação do paciente, e períodos mais curtos podem não fornecer uma estimativa adequada por conta da variação na ingestão diária ${ }^{10 .}$

Com relação ao consumo de alguns minerais e vitaminas, verifica-se que a ingestão

Tabela 4. Ingestão diária de energia e proteínas de pacientes submetidos a hemodiálise regular no Hospital das Clínicas. Recife, 2002.

\begin{tabular}{|c|c|c|c|c|c|c|c|}
\hline \multirow{2}{*}{$\underbrace{\text { Sexo }}_{\text {Nutrientes }}$} & \multicolumn{3}{|c|}{ Sexo Masculino } & \multicolumn{3}{|c|}{ Sexo Feminino } & \multirow{2}{*}{ Valor de $p^{* \star *}$} \\
\hline & $x$ & DP & $\%$ Adeq** & $x$ & DP & $\%$ Adeq** & \\
\hline Energia (kcal) & $1708,0 \pm$ & 362,0 & 92 & $1570,0 \pm$ & 452,0 & 93 & 0,867 \\
\hline Energia (kcal/kg de peso) & $31,0 \pm$ & 9,0 & - & $31,0 \pm$ & 9,0 & - & 0,922 \\
\hline Prot (g) & $77,0 \pm$ & 18,0 & 113 & $71,0 \pm$ & 19,0 & 113 & 0,344 \\
\hline Prot (g/kg de peso) & $1,4 \pm$ & 0,4 & - & $1,4 \pm$ & 0,5 & - & 0,896 \\
\hline Prot AVB* (g) & $50,0 \pm$ & 15,0 & - & $47,0 \pm$ & 17,0 & - & 0,562 \\
\hline$\%$ Prot AVB & 64,0 & & - & 65,0 & & - & 0,777 \\
\hline
\end{tabular}

* Prot AVB = Proteína de Alto Valor Biológico; ** $=\%$ de Adequação; *** Teste " $t$ " de Student. 
foi deficitária em cálcio e vitamina A (Tabela 5). Por outro lado, o consumo de fósforo foi além da recomendação, o que pode ocasionar alguns sérios problemas.

O fósforo deve ser restrito na dieta desses pacientes, pois os procedimentos dialíticos são pouco eficientes em sua remoção, podendo ocorrer hiperfosfatemia com todas as suas conseqüências ${ }^{7}$. Além disso, como a necessidade protéica é elevada, a ingestão de fósforo dificilmente será inferior a $800 \mathrm{mg} / \mathrm{dia}$, sendo, freqüentemente, necessário o uso de quelantes de fósforo. Por outro lado, os alimentos ricos em cálcio, como os laticínios, são também fontes de fósforo e, por esse motivo, rotineiramente são limitados na dieta, exigindo suplementação de cálcio.

Com base no exposto, pode-se pensar que o déficit na ingestão de cálcio teria como causa principal a orientação dietoterápica. No entanto, levantamentos realizados no Nordeste e no Sudeste do país, com diferentes grupos populacionais, evidenciaram a mesma magnitude de inadequação; ou seja, o déficit não estaria restrito a esse grupo de renais crônicos em particular ${ }^{29-31}$.

No que se refere à vitamina $A$, desde a década de 30 sabe-se da ocorrência de níveis séricos elevados de retinol em pacientes com IRC. No entanto, sintomas de toxicidade não foram relatados ${ }^{32}$. A teoria mais amplamente aceita para esse distúrbio é que, na insuficiência renal, a proteína carreadora do retinol (RBP) é menos catabolizada, sofrendo acúmulo, e tornando-se mais disponível para se ligar ao retinol, o qual é então retido na circulação ${ }^{33}$.

Por outro lado, vale a pena salientar que a grande maioria dos trabalhos, relatando a ocorrência de níveis séricos elevados de vitamina A na IRC, foi realizada em regiões sócioeconomicamente desenvolvidas onde, segundo Chazot \& Kopple $^{34}$, até mesmo as dietas hipoprotéicas, utilizadas em uma fase anterior à diálise, possuíam conteúdo normal de vitamina A. Realidade que, muito provavelmente, não corresponde à da grande maioria das regiões mais pobres.

Contextualizando o problema no Nordeste brasileiro, Kosminsky², em estudo realizado com pacientes em tratamento dialítico no Recife, Brasil, além de não encontrar níveis séricos elevados de retinol, constatou a presença de $8 \%$ de níveis abaixo da normalidade. Este fato novo no estudo do problema, levanta a questão da provável influência de condições socioeconômicas e demonstra a necessidade de maiores pesquisas na área, principalmente, no que se refere ao consumo alimentar e ao estado de saúde desse grupo sui-generis.

Neste estudo, o percentual de adequação dietética de vitamina A foi baixo, oscilando entre $51 \%$ e $65 \%$ (Tabela 5). Importa registrar que, na literatura especializada, há ausência de recomendações dietéticas de vitamina A para os pacientes com IRC. O que se encontra são contra-indicações para a suplementação. A

Tabela 5. Ingestão diária de cálcio, fósforo, ferro, zinco e vitamina A de pacientes submetidos a hemodiálise regular no Hospital das Clínicas. Recife, 2002.

\begin{tabular}{|c|c|c|c|c|c|c|c|c|}
\hline \multirow{2}{*}{ Sexo } & \multicolumn{4}{|c|}{ Sexo Masculino } & \multicolumn{3}{|c|}{ Sexo Feminino } & \multirow{2}{*}{ Valor de $p^{* *}$} \\
\hline & $x$ & & DP & $\%$ Adeq* & $x$ & DP & $\%$ Adeq* & \\
\hline Cálcio (g) & 440 & \pm & 222 & 44 & 685 & \pm 1053 & 45 & $0,362 * * *$ \\
\hline Fósforo (g) & 938 & \pm & 252 & 118 & 883 & $\pm \quad 332$ & 117 & 0,577 \\
\hline Ferro (mg) & 14 & \pm & 5 & 139 & 13 & \pm & 103 & 0,676 \\
\hline Zinco (mg) & 12 & \pm & 4 & 83 & 11 & \pm & 90 & 0,309 \\
\hline Vit $A(\mu R E)$ & 462 & \pm & 295 & 51 & 458 & 242 & 65 & 0,969 \\
\hline
\end{tabular}

* \% de Adequação; ** Teste " $\mathrm{t}$ " de Student ; *** Kruskal-Wallis. 
maioria dos textos técnicos não aborda a necessidade ou recomendação dietética. Portanto, neste estudo, utilizou-se como parâmetro a existente recomendação para adultos saudáveis ${ }^{13}$.

O baixo consumo de vitamina $A$ encontrado, pode ser um reflexo do que ocorre na população em geral. No Brasil, mais especificamente no Nordeste, o consumo dessa vitamina sempre foi deficitário. O Estudo Nacional de Despesa Familiar (ENDEF), realizado na década de 70, mostrou que, no Nordeste urbano, 53\% das famílias com crianças menores de 5 anos não consumiam nem a metade da recomendação diária dessa vitamina ${ }^{35}$. Mais recentemente, também da região Nordeste, outros pesquisadores encontraram incidências de consumo inadequado, da mesma ordem de grandeza que aqueles estimados pelo ENDEF, nos meados da década de $70^{30}$.

Os pacientes de ambos os sexos apresentaram uma ingestão de ferro adequada. No entanto, a deficiência desse elemento-traço é freqüente em pacientes com IRC, mesmo nas fases da doença que antecedem ao tratamento dialítico ${ }^{36}$. Essa condição é agravada em pacientes hemodialisados, nos quais podem ocorrer perdas significativas de sangue, seja durante o procedimento dialítico, ou em exames laboratoriais de rotina, em cirurgias e em acidentes com o acesso vascular $^{36,37}$. Essas perdas, se não forem repostas, podem levar a um balanço negativo de $1 \mathrm{~g}$ a $3 \mathrm{~g}$ de ferro por $a_{n}{ }^{37}$.

Outra dificuldade, em termos de manutenção adequada dos estoques de ferro, é a diminuição da absorção. A absorção desse micronutriente aumenta acentuadamente, em resposta a um quadro de deficiência. No entanto, em estudo conduzido por Kooistra et al. ${ }^{38}$, ficou evidenciado que, tanto a captação pela mucosa, quanto a retenção de ferro, foram significativamente diminuídas em pacientes submetidos a hemodiálise, quando comparados a indivíduos não-urêmicos deficientes em ferro.

A associação entre anemia e IRC é conhecida há mais de 150 anos, tendo como uma das principais causas a produção deficiente de eritropoietina (EPO) pelos rins. Em 1986, foram iniciados os ensaios terapêuticos com eritropoietina recombinante humana (Epo-rHu), tendo sido este um passo marcante no tratamento da anemia do paciente renal crônico ${ }^{36,37}$. No entanto, os estudos mostram que, se não houver estoques adequados de ferro, não haverá adequada hematopoiese. Ao mesmo tempo, as necessidades de ferro em pacientes recebendo Epo-rHu, encontram-se quase sempre acima da capacidade de absorção intestinal, indicando a necessidade de suplementação, geralmente através da via parenteral ${ }^{36}$.

Também foi proposta a suplementação com carnitina para melhorar os níveis de hemoglobina, sendo útil no tratamento da anemia resistente a eritropoietina ${ }^{39}$. No entanto, embora existam relatos de que essa suplementação possa ser efetiva no controle de muitos sintomas, tais como: astenia, cãibras intradialíticas, anorexia e hipotensão, a totalidade da evidência é insuficiente para recomendar o uso rotineiro da carnitina no tratamento dialítico ${ }^{7}$.

Doentes crônicos podem ainda manifestar carência de zinco, devido à diminuição da oferta alimentar ou ao aumento das perdas fecais e/ou urinárias, pois a IRC constitui uma das principais condições clínicas associadas à deficiência de zinco ${ }^{40}$.

No final da década de 80, com o objetivo de determinar as causas do metabolismo anormal de zinco na uremia, foi realizado um estudo de balanço de zinco em 10 pacientes adultos submetidos a hemodiálise e 5 controles normais. Os indivíduos foram alimentados com dieta padrão para hemodiálise, contendo 10mg de zinco. Após uma semana de estabilização, foram coletadas amostras de sangue, urina e fezes. Comparados aos controles, os pacientes tinham excreção urinária e níveis plasmáticos de zinco mais baixos e, ainda que as perdas dialíticas tenham sido mínimas, as perdas fecais foram elevadas; concluiu-se que tal resultado poderia ter como causa a má absorção de zinco, outra possível complicação da uremia ${ }^{41}$. 
De fato, Abu-Hamdan et al. ${ }^{42}$ já tinham evidenciado deficiência de absorção de zinco em pacientes dialisados, além de relatarem agravamento dessa condição com o uso de sulfato ferroso e quelantes de fósforo.

Alguns autores têm sugerido que a hipozincemia, em nefropatas crônicos pode estar relacionada à redistribuição do zinco corporal. Isso porque, na maioria dos estudos em que o zinco plasmático foi encontrado reduzido, os níveis nos eritrócitos estavam elevados ${ }^{43,44}$. Desse modo, não está claro se a hipozincemia na IRC representa uma verdadeira depleção de zinco corporal total ou uma mudança deste oligoelemento do compartimento extracelular para o intracelular ${ }^{44}$.

Segundo Martins ${ }^{7}$, na insuficiência renal, a deficiência de zinco pode estar ligada a muitos sintomas da doença, como a perda do apetite, redução do paladar (hipogeusia), do olfato (hiposmia) e distúrbios na função sexual. Apesar de alguns estudos sugerirem melhora desses sintomas com a suplementação de zinco ${ }^{45}$, outros estudos não confirmam esse achado ${ }^{46}$.

No presente estudo, a média de ingestão de zinco esteve dentro dos padrões esperados (Tabela 5). No entanto, esse fato não descarta a possibilidade de deficiência. Quanto aos possíveis benefícios da suplementação, Cabral \& Diniz ${ }^{40}$, em um trabalho de revisão sobre o tema, mostraram que os dados da literatura ainda não são conclusivos, muito embora, as evidências sugiram que o zinco venha a ter, futuramente, um papel importante no tratamento do paciente renal crônico, amenizando e/ou prevenindo muitos dos sintomas urêmicos.

\section{O N CLUSÃ O}

Com base nos resultados obtidos, conclui-se que, apesar das precárias condições socioeconômicas, a ingestão energético-protéica não foi deficitária, a média do IMC se situou dentro da faixa de normalidade e foi encontrada igual incidência de baixo peso e de excesso de peso entre os pacientes (18,9\% em cada caso) Por outro lado, o consumo de cálcio e vitamina A foi baixo ( $<50,0 \%$ e $<70,0 \%$ da ingestão diária recomendada, respectivamente), o que, principalmente para esse último nutriente, deixa clara a necessidade de pesquisas dentro do contexto social e regional. Se a dieta é deficiente em vitamina $A$, deve-se ter cautela ao tomar como padrão os resultados e as condutas praticadas em regiões desenvolvidas, onde as recomendações são baseadas no pressuposto de que os pacientes renais crônicos apresentam concentrações elevadas de retinol sérico.

\section{REFERÊ NCIAS}

1. Riella MC, Pecoits-Filho R. Insuficiência renal crônica: fisiopatologia da uremia. In: Riella MC. Princípios de nefrologia e distúrbios hidroeletrolíticos. 4.ed. Rio de Janeiro: Guanabara Koogan; 2003. p.661-90.

2. Lessa I. Outras doenças crônicas não transmissíveis de importância social. In: Lessa I. O adulto brasileiro e as doenças da modernidade: epidemiologia das doenças crônicas não transmissíveis. São Paulo: Hucitec; 1998. p.81-201.

3. Sociedade Brasileira de Nefrologia. Censo SBN 2002: dados consolidados por estado [Acesso em 15 dez. 2003]. Disponível em: http://www.sbn. org.br/

4. Sesso R. Inquérito epidemiológico em unidades de diálise no Brasil. J Bras Nefrol. 2000; 22(3 Supl 2):23-6.

5. Dwyer JT, Cunniff PJ, Maroni BJ, Kopple JD, Burrowes JD, Powers SN, et al. The hemodialysis pilot study: nutrition program and participant characteristics at baseline. J Ren Nutr. 1998; 8:(1)11-20.

6. Kopple JD. Pathophysiology of protein-energy wasting in chronic renal failure. J Nutr. 1999; 29 Suppl 1:247-51.

7. Martins C, Riella MC. Nutrição e Hemodiálise. In: Riella MC, Martins C. Nutrição e o rim. Rio de Janeiro: Guanabara Koogan; 2001. p.114-31. 
8. National Kidney Foundation: NKF-DOQI - I. Adult guidelines - A. Maintenance Dialysis - 1. Evaluation of protein-energy nutritional Status. New York: National Kidney Foundation; 2000.

9. World Health Organization. Physical status: The use and interpretation of anthropometry. Geneva; 1995. (WHO Technical Report Series, n.854).

10. National Kidney Foundation: NKF-DOQI - I. Adult guidelines, C. Appendices, Appendix III. Dietary interviews and diaries. New York: National Kidney Foundation; 2000.

11. Pinheiro AVB, Lacerda EMA, Haimbenzecry E, Gomes MCS, Costa VM. Tabela para avaliação de consumo alimentar em medidas caseiras. Rio de Janeiro; 1994. 74p. (Produção Independente).

12. Martins C. Vitaminas e oligoelementos na insuficiência renal. In: Riella MC, Martins C. Nutrição e o rim. Rio de Janeiro: Guanabara Koogan; 2001. p.43-57.

13. Institute of Medicine/Food and Nutrition Board Dietary Reference Intakes for vitamin A, vitamin K, arsenic, boron, chromium, copper, iodine, iron, manganese, molybdenum, nickel, silicon, vanadium and zinc. Washington, DC: National Academy Press; 2001.

14. World Health Organization. Epi Info. Version 6.04. A word processing, database and statistic program for public health [programa de computador]. Geneva; 1997.

15. Statistical Package for the Social Sciences for Windows. Student version. Release 7.5. Marketing Department. Chicago; 1996.

16. Programa de Apoio à Nutrição. Versão 2.5. Centro de Informática em Saúde da Escola Paulista de Medicina [software]. Universidade Federal de São Paulo. São Paulo; 1993.

17. Franco G. Tabela de composição química dos alimentos. São Paulo: Atheneu, 1997.

18. Lameire N. Management of the hemodialysis patient: an European perspective. In: Ronco C, Levin NW. Advances in end-stage renal disease; 2002. Basel: Karger; 2002. p.93-100.
19. Valenzuela RGV, Giffoni AG, Cuppari L, Canziani MEF. Estado nutricional de pacientes com insuficiência renal crônica em hemodiálise no Amazonas. Rev Assoc Med Bras. 2003; 49(1): 72-8.

20. Aparicio M, Cano N, Chauveau P, Azar R, Flory A, Laville $\mathrm{M}$, et al. Nutritional status of haemodialysis patients: a French national cooperative study. Nephrol Dial Transplant. 1999; 14(7):1679-86.

21. Jager KJ, Merkus MP, Huisman RM, Boeschoten EW, Dekker FW, Korevaar JC, et al. Nutritional status over time in hemodialysis and peritoneal dialysis. J Am Soc Nephrol. 2001; 12(6):1272-9.

22. Leavey SF, Strawderman RI, Jones CA, Port FK, Held PJ. Simple nutritional indicators as independent predictors of mortality in hemodialysis patients. Am J Kidney Dis. 1998; 31(6):997-1006.

23. Kopple JD, Zhu X, Lew NL, Lowrie EG. Body weight-for-height relationships predict mortality in maintenance hemodialysis patients. Kidney Int. 1999; 56(3):1136-48.

24. Canziani MEF, Draibe SA, Nadaletto MAJ. Técnicas dialíticas na insuficiência renal crônica. In: Ajzen H, Schor N. Nefrologia: guias de medicina ambulatorial e hospitalar. São Paulo: Manole; 2002. p.195-209.

25. Kuhlmann MK, Konig J, Riegel W, Kohler H. Gender-specific differences in dialysis quality (Kt/V): "big men" are at risk of inadequate haemodialysis treatment. Nephrol Dial Transplant. 1999; 14(1):147-53.

26. Lowrie EG, Lew NL. Death risk in hemodialysis patients: the predictive value of commonly measured variables and an evaluation of death rate differences between facilities. Am J Kidney Dis. 1990; 15(5):458-82.

27. Owen W, Lwe N, Lui Y, Lowrie EG, Lazarus, TM. The urea reduction ratio and serum albumin concentrations as predictors of mortality in patients undergoing hemodialysis. N Engl J Med. 1993; 329(14):1001-6.

28. Kaysen GA. Malnutrition and the acute-phase reaction in dialysis patients-how to measure and 
how to distinguish. Nephrol Dial Transplant. 2000; 15(10):1521-4.

29. Velásquez-Meléndez G, Martins IS, Cervato AM, Fornés NS, Marucci MFN. Consumo alimentar de vitaminas e minerais em adultos residentes em área metropolitana de São Paulo, Brasil. Rev Saude Publica. 1997; 31(2):157-62.

30. Albuquerque MFM, Monteiro AM. Ingestão de alimentos e adequação de nutrientes no final da infância. Rev Nutr. 2002; 15(3):291-9.

31. Cabral PC, Melo AMCA, Amado TCF, Santos RMAB. Avaliação antropométrica e dietética de hipertensos atendidos em ambulatório de um hospital universitário. Rev Nutr. 2003; 16(1): 61-71.

32. Kosminsky LB. Hipervitaminose A em pacientes em hemodiálise regular: um apriorismo [dissertação]. Recife: Universidade Federal de Pernambuco; 1993.

33. Muth I. Implication of hypervitaminosis A in chronic renal failure. J Ren Nutr. 1991; 1:2-8.

34. Chazot C, Kopple JD. Vitamin metabolism and requirements in renal disease and renal failure. In: Kopple JD, Massry SG. Nutritional management of renal disease. New York: Williams \& Wilkins; 1997. p.415-77.

35. Instituto Brasileiro de Geografia e Estatística. Perfil estatístico de crianças e mães no Brasil: aspectos nutricionais 1974-75. Rio de Janeiro; 1982.

36. Fishbane S, Maesaka JK. Iron management in end-stage renal disease. Am J Kidney Dis. 1997; 29(3):319-33.

37. Sakiewicz P, Paganini E. The use of iron in patients on chronic dialysis: mistake and misconceptions. J Nephrol. 1998; 11(1):5-15.
38. Kooistra M, Niemantsverdriet E, van Es A, Mol-Beermann N, Struyvenberg A, Marx J. Iron absorption in erythropoietin-treated haemodialysis patients; effects of iron availability, inflammation and aluminnium. Nephrol Dial Transplant. 1998; 13(1):82-8.

39. Ahmad S, Hoppel C, Nava D, Nicora R. Role of L-carnitine in treating renal dialysis patients. Dial Transplant. 1994; 23:177-81.

40. Cabral PC, Diniz AS. Alterações metabólicas e funcionais do zinco em pacientes com insuficiência renal crônica. Rev Bras Nutr Clin. 2003; 18(1): 31-6.

41. Mahajan SK. Factors underlying abnormal zinc metabolism in uremia. Kidney Int. 1989; 36(275): 5269-73.

42. Abu-Hamdan DK, Mahajan SK, Migdal SD, Prasad AS, McDonald FD. Zinc tolerance test in uremia. Ann Intern Med. 1986; 104(1):50-2.

43. Smythe WR, Alfrey AC, Craswell PW, Crouch CA, Ibels LS, Kubo H, et al. Trace element abnormalities in chronic uremia. Ann Int Med. 1982; 96(3): 302-10.

44. Kimmel PL, Watkins DH, Teller EB, Khanna R, Dosa $S$, Phillips TM. Zinc balance in combined zinc deficiency and uremia. Kidney Int. 1988; 33(6):1091.

45. Antoniou LD, Shalhoud RJ. Zinc -induced enhancement of lymphocyte function and viability in chronic uremia. Nephron. 1985; 40(1):13-21.

46. Bonomini M, Di Paolo B, De Risio F, Niri L, Klinkmann $\mathrm{H}$, Ivanovich $\mathrm{P}$, et al. Effects of zinc supplementation in chronic haemodialysis patients. Nephrol Dial Transplant. 1993; 8(10): 1166-8.

Recebido para publicação em 12 de maio de 2003 e aceito em 3 de março de 2004. 